\title{
Extended collimator model for pencil-beam dose calculation in proton radiotherapy
}

\author{
Nobuyuki Kanematsu ${ }^{1}$, Takashi Akagi ${ }^{2}$, \\ Yasuyuki Takatani ${ }^{3}$, Shunsuke Yonai ${ }^{1}$, \\ Hidenobu Sakamoto ${ }^{3}$ and Haruo Yamashita ${ }^{4}$ \\ 1 National Institute of Radiological Sciences, 4-9-1 Anagawa, Inage-ku, Chiba \\ 263-8555, Japan \\ 2 Hyogo Ion Beam Medical Center, 1-2-1 Kouto, Tatsuno, Hyogo 679-5165, \\ Japan \\ 3 Mitsubishi Electric Corporation, 1-1-2 Wadasakicho, Hyogo-ku, Kobe, Hyogo \\ 652-8555, Japan \\ 4 Shizuoka Cancer Center, 1007 Shimonagakubo, Nagaizumi-machi, Suntou-gun, \\ Shizuoka 411-8777, Japan \\ E-mail: nkanemat@nirs.go.jp
}

\begin{abstract}
We have developed a simple collimator model to improve the accuracy of penumbra behaviour in pencil-beam dose calculation for proton radiotherapy. In this model, transmission of particles through a threedimensionally extended opening of a collimator is calculated in conjunction with phase space distribution of the particles. Comparison of the dose distributions calculated using the new three-dimensional collimator model and the conventional two-dimensional model to lateral dose profiles experimentally measured with collimated proton beams showed the superiority of the new model over the conventional one.
\end{abstract}

PACS numbers: 87.53.Pb, 87.53.Tf, 87.53.Uv

Submitted to: Phys. Med. Biol.

\section{Introduction}

The pencil-beam algorithm with the Gaussian beam model, which was originally developed for dose calculation in electron radiotherapy (Brahme et al 1981, Hogstrom et al 1981 and Jette 1988), is now widely used in proton radiotherapy to handle spatial beam modulation due to heterogeneity (Petti 1992, Hong et al 1996, Carlsson et al 1997, Schaffner et al 1999, Russell et al 2000, Szymanowski et al 2001 and Hollmark et al 2004). In the pencil-beam algorithm for collimated broad beams, a collimator is usually modelled as an infinitesimally thin and complete shield with a two-dimensionally (2D) shaped opening. The 2D collimator model has been developed and validated for aperture blocks made of heavy metal with blocking thickness of less than several centimetres (Hong et al 1996).

Recently, Hyogo Ion Beam Medical Center (HIBMC) and Shizuoka Cancer Center (SCC) started proton radiotherapy with similar rotating gantries. Their beam delivery 
systems form uniform broad beams of source-axis distance (SAD) approximately $3 \mathrm{~m}$ with magnetic beam wobbling (Akagi et al 2003). The multileaf collimators (MLC's) shape the fields conformal to given targets with straight-ended iron leaves of blocking thickness of $14 \mathrm{~cm}$, which is more than twice the maximum beam range to prevent from penetration through the stepped leaf gaps (Chu et al 1993). Though the 2D collimator model for a similarly structured MLC has been validated for certain carbonion beams (Kohno et al 2004), the validity is not warranted for the proton beams. The proton gantries have a relatively short $\mathrm{SAD}$, resulting in larger beam divergence, and protons receive larger scattering than carbon ions by nature, resulting in larger beam emittance. These differences will enhance the effect of the collimator thickness, which has been ignored in the $2 \mathrm{D}$ model.

Detailed dosimetric behaviours of proton beams could be estimated empirically by measurement (Oozeer et al 1997), or theoretically by Monte Carlo simulation (Paganetti 1998). However, the empirical approach would require a great deal of measurement, parameterisation and data management to prepare for all the possible beam customisations required for treatment. On the other hand, use of the Monte Carlo method has been limited to quality assurance of beam delivery systems (Paganetti et al 2004) and validation of sampled plans (Jiang and Paganetti 2004) rather than for treatment planning because of impractical calculation time.

We have thus improved the pencil-beam dose calculation in the treatment planning system at HIBMC and SCC (Kanematsu et al 1998 and Akagi et al 2006) by taking three-dimensional (3D) collimator structures into account to reflect their effect in individual treatment plans. In this paper, we first introduce our implementation of the pencil-beam algorithm, then present the new 3D collimator model and finally evaluate its accuracy and necessity through comparisons with the conventional $2 \mathrm{D}$ model and measurement in penumbra behaviour of a proton beam with several collimated fields.

\section{Materials and methods}

\subsection{The pencil-beam algorithm}

In the pencil-beam algorithm, a broad beam is virtually decomposed to small pencil beams, which develop individually in the heterogeneous medium. Dose distribution $D_{i}(\vec{r})$ from pencil beam $i$ is sequentially calculated and accumulated to form total dose distribution $D(\vec{r})$,

$$
D(\vec{r})=\sum_{i \in \text { vicinity }} D_{i}(\vec{r})
$$

where the summation is taken over the pencil beams in the vicinity of calculation point $\vec{r}$, which are expected to make certain dose contribution to the point.

The model representation depends on implementation and, in this case (Kanematsu et al 1998), pencil beam $i$ is described with residual range $R_{i}(s)$, projected angular variance ${\overline{\theta^{2}}}_{i}(s)$, projected angular-spatial covariance $\overline{t \theta}_{i}(s)$ and projected spatial variance $\bar{t}^{2} i(s)$ of the involved particles, where $t$ and $\theta$ represent the transverse position and the angle of the particle, respectively, as a function of path length along the central axis $s$, as shown in figure 1. The pencil beam generated on the exit face of the collimator $(s=0)$ develops in beam transport through matter using

$$
\overline{t \theta}_{i}(s)=\overline{t \theta}_{i}(0)+s{\overline{\theta^{2}}}_{i}(0)+\int_{0}^{s}\left(s-s^{\prime}\right) \frac{\mathrm{d} \bar{\theta}_{i}\left(s^{\prime}\right)}{\mathrm{d} s^{\prime}} \mathrm{d} s^{\prime}
$$




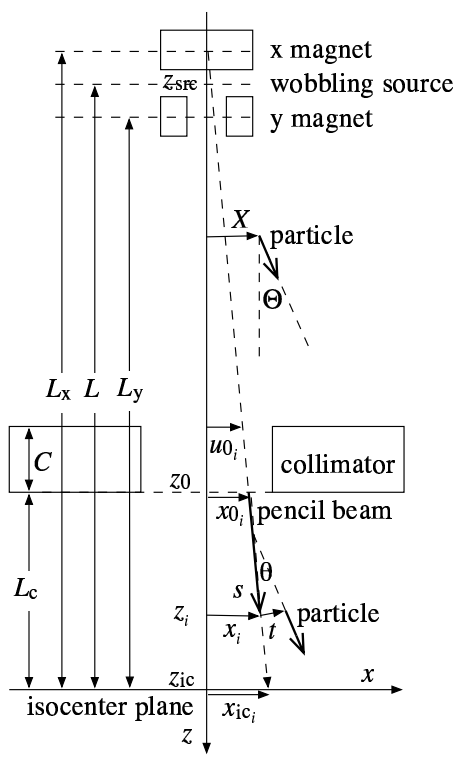

Figure 1. Definition of geometric parameters.

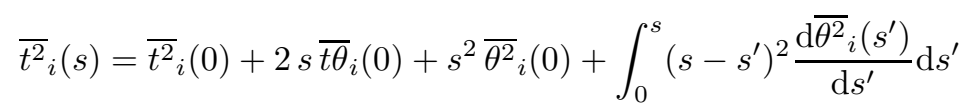

in the Fermi-Eyges theory (Tomura et al 1998 and Hollmark et al 2004). Scattering power $\mathrm{d} \bar{\theta}^{2}{ }_{i} / \mathrm{d} s$ is calculated using the Highland formula for multiple Coulomb scattering in heterogeneous medium (Gottshalk et al 1993),

$$
\frac{\mathrm{d} \bar{\theta}_{i}^{2}(s)}{\mathrm{d} s}=\frac{14.1^{2}}{p^{2} \beta^{2}} \frac{\rho\left(\overrightarrow{p_{i}}(s)\right)}{w_{R}\left(\overrightarrow{p_{i}}(s)\right)}\left[1+\frac{1}{9} \log _{10}\left(\int_{0}^{s} \frac{\rho\left(\overrightarrow{p_{i}}\left(s^{\prime}\right)\right)}{w_{R}\left(\overrightarrow{p_{i}}\left(s^{\prime}\right)\right)} \mathrm{d} s^{\prime}\right)\right]^{2}
$$

where $\rho$ and $w_{R}$ are the density in $\mathrm{g} / \mathrm{cm}^{3}$ and the radiation length in $\mathrm{g} / \mathrm{cm}^{2}$, respectively, of the material. The pencil-beam path is represented by centre position $\vec{p}_{i}=\left(x_{i}, y_{i}, z_{i}\right)$ as a function of path length $s, \overrightarrow{p_{i}}(s)=\vec{p}_{0_{i}}+\left(\mathrm{d} \overrightarrow{p_{i}} / \mathrm{d} s\right) s$, with origin $\overrightarrow{p_{0}}=\left(x_{0 i}, y_{0_{i}}, z_{0}\right)$ on the collimator exit face and direction $\mathrm{d} \overrightarrow{p_{i}} / \mathrm{d} s$. Proton momentum $p$ in $\mathrm{MeV} / c$ and velocity $\beta$ in $c$ are given by the proton energy converted with the range-energy relationship (ICRU 1993) and residual range $R_{i}$ is related to waterequivalent depth,

$$
w_{i}(s)=R_{0}-R_{i}(s)=\int_{0}^{s} \frac{S\left(\overrightarrow{p_{i}}\left(s^{\prime}\right), \beta\right)}{S_{\text {water }}(\beta)} \mathrm{d} s^{\prime} \simeq \int_{0}^{s} \rho\left(\overrightarrow{p_{i}}\left(s^{\prime}\right)\right) \mathrm{d} s^{\prime}
$$

where residual range at the origin $R_{0}$ is common to all the pencil beams. Since the $\beta$ dependence predominantly cancels out, stopping power ratio $S / S_{\text {water }}$ is approximated to effective density $\rho$, which is measured by planning x-ray CT with appropriate $\mathrm{CT}$ number conversion (Kanematsu et al 2003).

Since the pencil beams are generated in infinitesimal size, we approximate initial values as $\bar{t}^{2}{ }_{i}(0) \simeq \overline{t \theta}_{i}(0) \simeq 0$. Direction $\mathrm{d} \vec{p}_{i} / \mathrm{d} s$ and angular variance ${\overline{\theta^{2}}}_{i}(0)$, which describe the phase-space distribution on the pencil-beam generation plane, are related to the characteristics of the broad beam formed by the upstream beam-modifying devices. The method to obtain the phase-space distribution for a treatment beam 

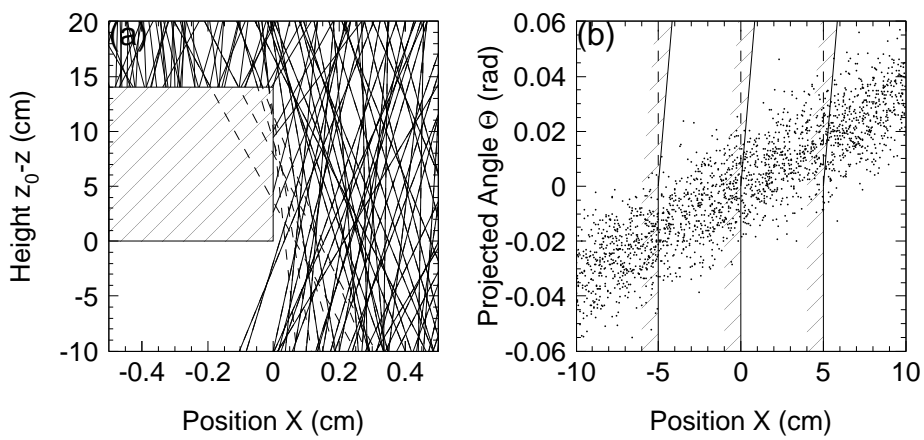

Figure 2. Example of broad beam collimation in simple Monte Carlo simulation with $L_{\mathrm{x}}-L_{\mathrm{c}}=3 \mathrm{~m}, C=14 \mathrm{~cm}$ and Gaussian angular spread $\sqrt{ }\left(\bar{\Theta}^{2}-\bar{\Theta}^{2}\right)=10$ mrad: (a) Side view of the left collimator positioned at $0 \mathrm{~cm}$ (hatched box) and the particle tracks incident from above (lines). (b) Phase space plot with dots representing the particles distributed in position and angle on the collimator exit face. The hatched sides of the solid lines are shadowed by the left collimator at retracted $-5 \mathrm{~cm}, 0 \mathrm{~cm}$ and extracted $+5 \mathrm{~cm}$ while the vertical dashed lines show the corresponding edges in the $2 \mathrm{D}$ model.

depends on beam delivery method and algorithm implementation. In our case, an analytic model of the beam-wobbling system (Tomura et al 1998) gives the phasespace distribution as described in Appendix A.

The dose contribution at point $\vec{r}=(x, y, z)$ from pencil beam $i$ is given by the product of broad-beam depth-dose distribution $D_{B B}(w)$ measured at the isocentre, the Gaussian line-spread function and geometric correction factors,

$$
D_{i}(\vec{r})=\frac{\Phi_{\mathrm{ic}}\left(r_{\mathrm{ic} i}\right)}{\Phi_{\mathrm{ic}}(0)} T_{i} \frac{\delta_{x} \delta_{y}}{\epsilon} D_{\mathrm{BB}}\left(w_{i}\left(s_{r}\right)\right) \frac{1}{2 \pi \overline{t^{2}} i\left(s_{r}\right)} e^{-\frac{\left|\vec{r}-\overrightarrow{p_{i}}\left(s_{r}\right)\right|^{2}}{2 \bar{t}^{2} i\left(s_{r}\right)}}
$$

where path length $s_{r}=\left(\mathrm{d} \overrightarrow{p_{i}} / \mathrm{d} s\right) \cdot\left(\vec{r}-\overrightarrow{p_{0}}\right)$ leads point $\vec{r}$ to be on the transverse plane, off-axis fluence ratio $\Phi_{\mathrm{ic}}\left(r_{\mathrm{ic} i}\right) / \Phi_{\mathrm{ic}}(0)$, given in Appendix A, reflects the fluence of the broad beam, pencil-beam intervals $\delta_{x}$ and $\delta_{y}$, typically a few millimetres at the isocentre, corrects for the areal number density of the pencil beams and collection efficiency $\epsilon=0.966$ corrects for the acceptance of vicinity condition $\left|\vec{r}-\overrightarrow{p_{i}}\left(s_{r}\right)\right|^{2}<$ $3 \bar{t}^{2}\left(s_{r}\right)$ applied in (1).

In the conventional 2D model, transmission factor $T_{i}=T_{2 \mathrm{D}}\left(x_{0 i}, y_{0 i}\right)$ takes binary value of either 1 for complete transmission or 0 for complete shielding when $\left(x_{0 i}, y_{0 i}\right)$ is in or out of, respectively, the collimator opening on the exit face.

\subsection{The $3 D$ extended collimator model}

In proton radiotherapy, either a non-divergent aperture block or a straight-ended MLC, having constant opening shape from entrance through exit, is usually used. Figure 2 shows an example of the effect of such a three-dimensionally extended collimator in relation to position-angle phase space of incident particles. Some of the incident particles hit the collimator on the entrance face though they are pointing to the opening on the exit face as shown as the dashed lines in figure 2(a). Three collimator positions are shown in figure 2(b), where the dots between the solid and the 
dashed lines represent the particles additionally blocked by the extended collimator. While the phase-space restriction is position independent, the correlation between the particle position and the incident angle, namely beam divergence, makes the effect of the phase-space restriction dependent on the collimator position.

We simply ignore these particles hitting the collimator assuming that the majority of them will stop in the collimator or are insignificant to the dose distribution. A Monte Carlo study showed that particles scattered at the edge would lose large energy in the collimator (van Luijk et al 2001), to cause large range shortening. Therefore, the edgescattered particles may be reasonably neglected since we are especially interested in the dose distribution in the treatment target located near the original beam range.

Back projection of pencil beam $i$ onto the entrance face of the collimator of thickness $C$ using equations (3) and (A.1) leads the involved particles to distribute in space $(u, v)$ with projected spatial variance $C^{2} \bar{\theta}^{2}{ }_{i}$ around centre $\left(u_{0 i}, v_{0 i}\right)$ with $u_{0 i}=x_{0 i}-x_{0 i} C /\left(L_{\mathrm{x}}-L_{\mathrm{c}}\right)$ and $v_{0 i}=y_{0 i}-y_{0 i} C /\left(L_{\mathrm{y}}-L_{\mathrm{c}}\right)$. Requiring for these particles to enter the opening on the entrance face in addition to the exit face, transmission factor $T_{i}$ is modified to

$$
T_{i}=T_{2 \mathrm{D}}\left(x_{0 i}, y_{0 i}\right) \frac{1}{2 \pi C^{2} \bar{\theta}_{i}^{2}} \iint T_{2 \mathrm{D}}(u, v) e^{-\frac{\left(u-u_{0 i}\right)^{2}+\left(v-v_{0 i}\right)^{2}}{2 C^{2} \bar{\theta}_{i}}} \mathrm{~d} u \mathrm{~d} v
$$

where factor $T_{2 \mathrm{D}}\left(x_{0 i}, y_{0 i}\right)$ requires that pencil beam $i$ is in the opening on the exit face and the integral gives the probability of particles with the 2D Gaussian spatial distribution to enter the same opening represented by $T_{2 \mathrm{D}}(u, v)$ on the entrance face.

Here, we have ignored occasions such that the particles hit the edge of the opening even though they enter the opening on both extreme faces, which may happen in case of a very irregular field. The irregularity is predominantly determined by the projected target shape and is characterized by its curvature. The typical radius of curvature for fields with conventional margining will be order of centimetres while shifts $x_{0 i} C /\left(L_{\mathrm{x}}-L_{\mathrm{c}}\right), y_{0 i} C /\left(L_{\mathrm{y}}-L_{\mathrm{c}}\right)$ and spread $C \sqrt{ }\left(\overline{\theta^{2}}{ }_{i}\right)$ of the pencil beam travelled through the collimator are typically less than a few millimetres. Therefore, the field edge within the spread of the pencil beam may usually be regular enough to justify this approximation.

In addition to the transmission factor, the phase-space restriction will deform the angular distribution of the pencil beam. An example is shown in figure 3(a), where a pencil beam entering near a collimator edge are partially blocked and is bent outward from the field. This is because, among the incident particles, only those with large angle inward to the field are blocked by the collimator. To best approximate the deformed angular distribution of the particles with the Gaussian function, schematically shown as the solid line in figure $3(\mathrm{~b})$, the pencil beam direction and the initial angular variance about the $s$-axis are redefined using the transmitted particles.

$$
\begin{aligned}
& \frac{\mathrm{d} x_{i}^{\prime}}{\mathrm{d} z_{i}}=\frac{1}{T_{i}} \frac{1}{2 \pi C^{2} \bar{\theta}_{i}^{2}} \iint T_{2 \mathrm{D}}(u, v) \frac{x_{0 i}-u}{C} e^{-\frac{\left(u-u_{0 i}\right)^{2}+\left(v-v_{0 i}\right)^{2}}{2 C^{2}{\overline{\theta^{2}}}_{i}}} \mathrm{~d} u \mathrm{~d} v \\
& \frac{\mathrm{d} y_{i}^{\prime}}{\mathrm{d} z_{i}}=\frac{1}{T_{i}} \frac{1}{2 \pi C^{2} \bar{\theta}^{2}} \iint T_{2 \mathrm{D}}(u, v) \frac{y_{0 i}-v}{C} e^{-\frac{\left(u-u_{0}\right)^{2}+\left(v-v_{0}\right)^{2}}{2 C^{2} \bar{\theta}^{2}}} \mathrm{~d} u \mathrm{~d} v
\end{aligned}
$$



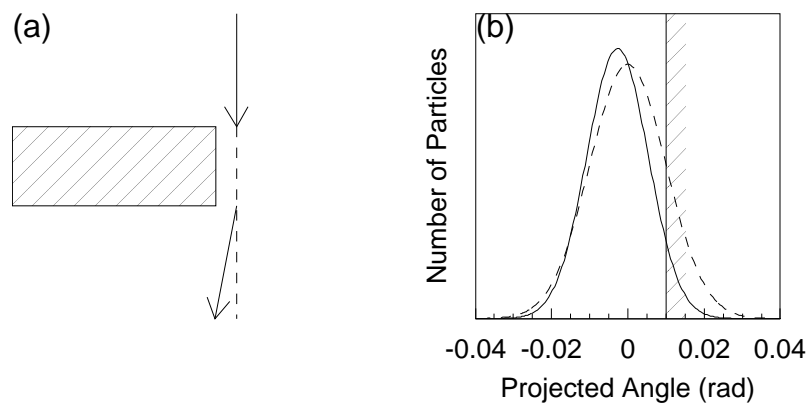

Figure 3. Example of phase-space restriction for a pencil beam near a left collimator edge; (a) side view of the collimator (hatched box) and incoming and transmitted pencil beams (arrows) and (b) projected angle distribution for the incoming pencil beam (dashed line), where the particles in the hatched side region are blocked by the collimator, and that for the transmitted pencil beam (solid line).

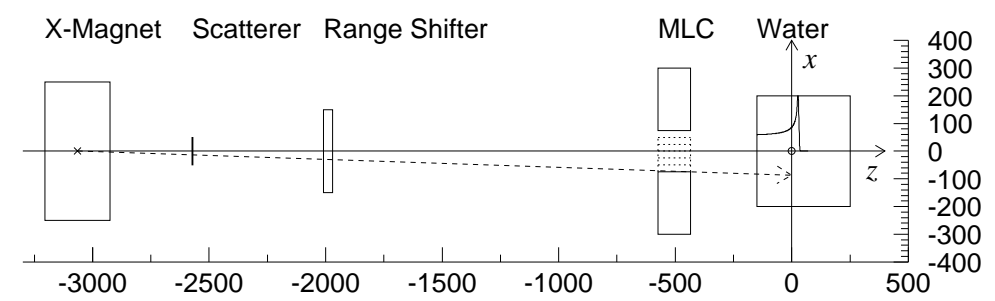

Figure 4. Experimental apparatus at HIBMC with dimensions in millimetres. The $\mathrm{X}$ - and the o-marks indicate the $x$-bending source and the isocentre, respectively. The dashed arrow from the source points to the field edge defined in section 3. The $y$-bending magnet and the beam monitors in the proton gantry are not shown here.

$$
\begin{aligned}
& {\overline{\theta^{2}}}_{i}^{\prime} \simeq \frac{1}{T_{i}} \frac{1}{2 \pi C^{2} \bar{\theta}^{2}} \iint\left\{T _ { 2 \mathrm { D } } ( u , v ) \frac { 1 } { 2 } \left[\left(\frac{x_{0 i}-u}{C}-\frac{\mathrm{d} x_{i}}{\mathrm{~d} z_{i}}\right)^{2}\right.\right. \\
& \left.\left.+\left(\frac{y_{0 i}-v}{C}-\frac{\mathrm{d} y_{i}}{\mathrm{~d} z_{i}}\right)^{2}\right] e^{-\frac{\left(u-u_{0 i}\right)^{2}+\left(v-v_{0 i}\right)^{2}}{2 C^{2} \bar{\theta}^{2}}}\right\} \mathrm{d} u \mathrm{~d} v
\end{aligned}
$$

Direction $\mathrm{d} \vec{p}_{i}^{\prime} / \mathrm{d} z_{i}=\left(\mathrm{d} x_{i}^{\prime} / \mathrm{d} z_{i}, \mathrm{~d} y_{i}^{\prime} / \mathrm{d} z_{i}, 1\right)$ replaces the pencil-beam direction, $\mathrm{d} \overrightarrow{p_{i}} / \mathrm{d} z_{i}$, and variance ${\overline{\theta^{2}}}_{i}^{\prime}$ replaces the initial angular variance, ${\overline{\theta^{2}}}_{i}(0)$, in pencil-beam transport (2)-(5).

In the numerical calculation, the integrals in (7)-(10) are done by summation over the pencil beam generation grids, where we apply vicinity condition $\sqrt{ }\left[\left(u-u_{0 i}\right)^{2}+\left(v-v_{0 i}\right)^{2}\right]<3 C^{2} \sqrt{ }\left(\bar{\theta}^{2}{ }_{i}\right)$ with collection-efficiency correction 1/0.966 in analogy with summation (1). The increase of calculation time for the 3D transmission calculation was typically $15 \%$ of the dose calculation time, which is in the range of seconds to minutes depending on volume and resolution clinically required. 


\subsection{Experimental apparatus}

We compared the 2D model and the 3D model calculations against measurement at HIBMC in the apparatus shown in figure 4, where the wobbling radius on the isocetre plane, the scatterer and the range shifter were set to $107 \mathrm{~mm}, 2.5-\mathrm{mm}$ lead and 40mm polyethylene, respectively. We took a $190-\mathrm{MeV}$ proton beam as an example of the typical treatment beams except that range modulation to form spread-out Bragg peak was not applied for simplicity. Since the range modulation is superposition of beams of different incident energies, the discussion with this example will basically apply to more general cases. We examined several MLC fields, where all the right leaves were fixed to the fully retracted position $(+75 \mathrm{~mm})$ and all the left leaves were coherently set, just like a collimator jaw, at several positions with mechanical precision within $\pm 0.5 \mathrm{~mm}$.

For those fields, we measured lateral dose profiles on the isocentre plane by scanning a $15-\mathrm{mm}^{3} 2-\mathrm{mm} \phi$ pinpoint ionization chamber at minimum 1-mm interval. The measurement depth was chosen to $150 \mathrm{~mm}$ from the water surface or $28 \mathrm{~mm}$ upstream of the distal $80 \%$-dose depth, which is to represent the target depth since the beam range will be adjusted to the maximum depth of the target. For dose output comparison among the experiment and the calculations, the monitor unit was calibrated against the isocentre dose of the reference field with the fully retracted $( \pm 75$ $\mathrm{mm}$ ) collimator opening.

The mechanical precision and the dose precision of the measurement system were within $\pm 0.5 \mathrm{~mm}$ and $\pm 1 \%$ of the maximum dose, respectively, based on the reproducibility analysis. The effective detector size for the $2-\mathrm{mm} \phi$ cylindrical chamber is geometrically estimated from the ionization track length profile for an infinitesimal beam to be $\sqrt{ }\left[\int_{-1}^{1} x^{2} \sqrt{ }\left(1-x^{2}\right) \mathrm{d} x / \int_{-1}^{1} \sqrt{ }\left(1-x^{2}\right) \mathrm{d} x\right]=0.5 \mathrm{~mm}$. Therefore, we neglect all the spatial and dosimetric errors in analysis of 1-mm resolution.

\section{Results and discussion}

Figure 5 shows the resultant dose profiles for the examined fields, where we define the field edge as the position of the left-collimator edge on the exit face projected from the bending source to the isocentre plane. The $2 \mathrm{D}$ model has been designed to produce sigmoid curves of the error function centred at the field edges, which are actually verified here. We observed substantial dose enhancement at shoulders and tails in some of the measured penumbra behaviours, which could be explained as additional dose contributions from edge scattering, though ignored in the calculation.

For the fields with the collimator at retracted positions $(-75 \mathrm{~mm},-50$ $\mathrm{mm}$ and $-25 \mathrm{~mm}$ ), the two calculations agreed well with each other and also with the measurement. However, for the fields at zero and modestly extracted collimator positions $(0 \mathrm{~mm}$ and $+25 \mathrm{~mm})$, the $2 \mathrm{D}$-model calculation departs from the measurement. In contrast, the transmission suppression for the pencil beams near the field edge in the 3D-model calculation reproduces the gentler penumbra shoulders as observed in the measurement.

For the field at the fully extracted collimator position $(+50 \mathrm{~mm})$, the 3Dmodel calculation and the 2D-model calculation are both in disagreement with the measurement in opposite directions. The underdose in the 3D-model calculation could be explained also by the ignored contribution from edge scattering. The offcentred field (left at $+50 \mathrm{~mm}$ and right at $+75 \mathrm{~mm}$ ) requires the particles to have 

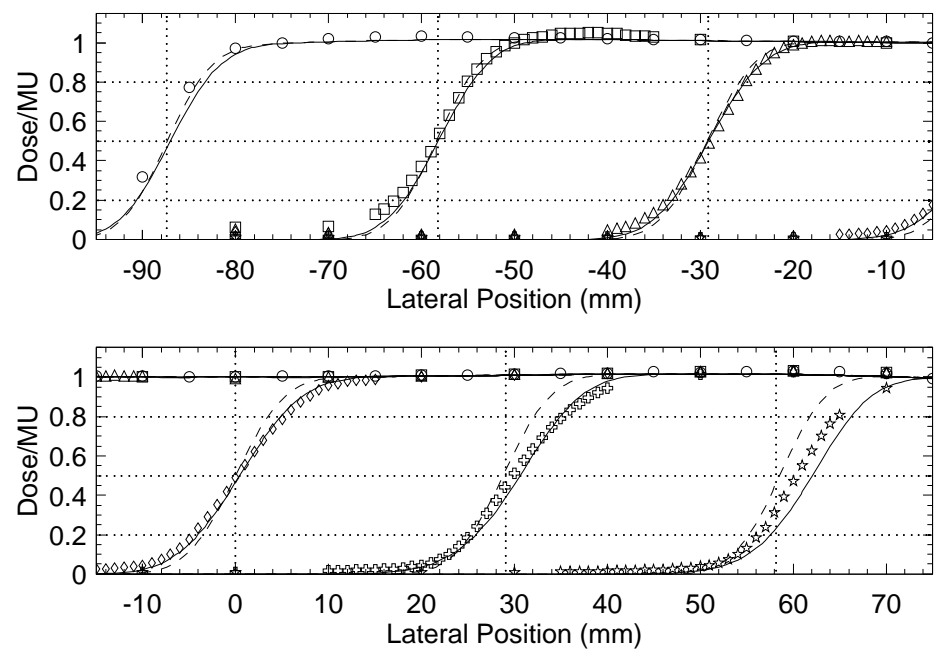

Figure 5. Lateral dose profiles on the isocentre plane for the examined fields. The marker points show the measured doses for the fields with the left collimator at $-75 \mathrm{~mm}$ (circles), $-50 \mathrm{~mm}$ (squares) $-25 \mathrm{~mm}$ (triangles), $0 \mathrm{~mm}$ (diamonds), $+25 \mathrm{~mm}$ (crosses) and $+50 \mathrm{~mm}$ (stars). The solid lines and the dashed lines show the dose profiles calculated with the $3 \mathrm{D}$ model and the $2 \mathrm{D}$ model, respectively. The vertical dotted lines indicate the field edges and the horizontal ones indicate the $20 \%$-, $50 \%$ - and $80 \%$-dose levels.
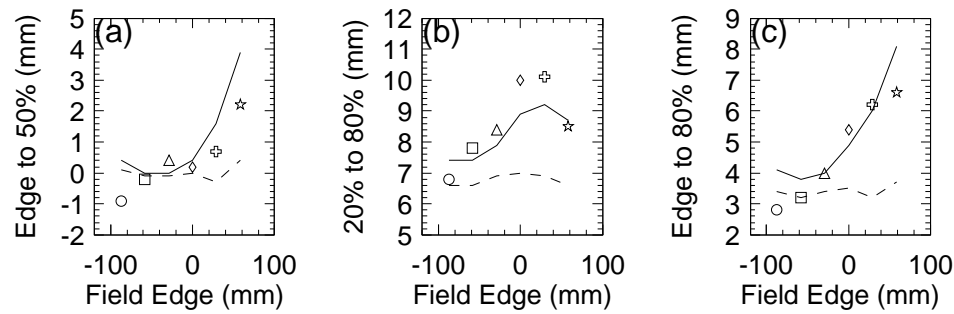

Figure 6. (a) 50\%-dose position with respect to the field edge, (b) penumbra size and (c) $80 \%$-dose position with respect to the field edge, as a function of field edge position. The markers, the solid and the dashed lines represent the measurement, the $3 \mathrm{D}$-model and the 2D-model calculations, respectively.

large angles and, thus, leads to a higher probability of edge scattering. In addition, the small opening width of $25 \mathrm{~mm}$ implies that not only the particles scattered at the left collimator but also those scattered at the right collimator may have largely contributed.

In figure 6 , we have plotted measures of penumbra behaviour for the measured and the calculated dose profiles in figure 5 , as a function of the field-edge position. Figure 6 (a) shows the 50\%-dose position, which ideally coincides with the field edge in the $2 \mathrm{D}$ model, with respect to the field edge position and figure 6(b) shows the penumbra size defined as the distance between $80 \%$ - and $20 \%$-dose points, which may be the most popular measure of the field edge blurring. In these plots, the 2D- 
model calculation leads as expected to nearly constant values within the calculation precision of a fraction of a millimetre. In contrast, the 3D-model calculation, as well as the measurement, leads to asymmetric dependence on the field-edge position. The penumbra size for the 3D model is at maximum 30\% larger than that for the $2 \mathrm{D}$ model, implying that the quadratic contribution of the MLC transmission effect is comparable to that of the intrinsic beam emittance. Since the transmission effect strongly increases with the collimator thickness according to (7), the 2D model may still be a good approximation for the aperture blocks with thickness up to several centimetres, with which the beam emittance will dominate the penumbra.

The measured dose profiles in figure 5 suggest existence of relatively large contribution from edge scattering at the $20 \%$-dose positions. Therefore, the penumbra size using the $20 \%$-dose position may not be the best measure of the beam modelling without care for the edge scattering. In addition, since the field should be formed to precisely cover the target volume with the prescribed dose in treatment planning, the accuracy in the shoulder of the penumbra is clinically more important than the penumbra size. Figure $6(\mathrm{c})$ shows the $80 \%$-dose position with respect to the field edge, which is to measure the penumbra shoulder. Good agreement of the 3D-model calculation with the measurement validates the implemented transmission model. In contrast, the 2D model resulted in discrepancy up to a few millimetres, which could be problematic to some critical cases in high-precision radiotherapy with proton beams, where orthogonal x-ray image-guided patient positioning against digitally reconstructed radiographs has been routinely practised (Suit H and Urie M 1992).

For a thick collimator system such as the MLC of this study, the penumbra behaviour varies with the field-edge position and thus should be examined with various fields in calibrating and/or validating a dose calculation system. Furthermore, optimisation of the hardware structure may be desirable in a similar manner as for the photon MLC's (Huq et al 2002) to minimise the position-dependent variation. The presented 3D collimator model is generally applicable to dose calculation systems of the pencil-beam algorithm including ones for heavy-ion radiotherapy and will be especially effective for compact machines of the near future (Komori et al 2004) for the same reason as with the proton gantries.

\section{Conclusions}

We have measured the penumbra behaviour of a proton beam with a thick collimator and revealed that the penumbra depends on the field-edge position and becomes gradual when the collimator is extracted beyond the centre line, which can not be reproduced by the conventional $2 \mathrm{D}$ collimator model.

The 3D extended collimator model, with which the transmission factor, the direction and the angular variance of each pencil beam are modified by the statistical calculation using the phase-space distribution of the particles, improves accuracy of the penumbra behaviour, especially in the shoulder part that determines the target dose coverage in treatment planning.

\section{Appendix A. Analytic model for beam wobbling}

The beam-wobbling system, consisting of a pair of orthogonally bending magnets, rotates the scattered beam circularly on the isocentre plane to form uniform field and 
the pencil beams are approximated to virtually radiate from the magnets, which leads the pencil beam direction to,

$$
\frac{\mathrm{d} \vec{p}_{i}}{\mathrm{~d} z_{i}}=\left(\frac{x_{0 i}}{L_{\mathrm{x}}-L_{\mathrm{c}}}, \frac{y_{0 i}}{L_{\mathrm{y}}-L_{\mathrm{c}}}, 1\right)
$$

where, $L_{\mathrm{x}}, L_{\mathrm{y}}$ and $L_{\mathrm{c}}$ are the distances of the $\mathrm{x}$ magnet, the y magnet and the exit face of the collimator from the isocentre, respectively, as shown in figure 1.

With the point-source approximation for beam wobbling, where the wobbling source is defined by SAD $L=\sqrt{ }\left(L_{\mathrm{x}} L_{\mathrm{y}}\right)$, Tomura et al (1998) derived analytic solutions for fluence $\Phi_{\mathrm{ic}}$ and projected angular variance $\overline{\theta_{\mathrm{ic}}{ }^{2}}$ of particles at off-axis distance $r_{\mathrm{ic}}=\sqrt{ }\left(x_{\mathrm{ic}}^{2}+y_{\mathrm{ic}}^{2}\right)$ on the isocentre plane $\left(z=z_{\mathrm{ic}}\right)$ as,

$$
\begin{aligned}
\Phi_{\mathrm{ic}}\left(r_{\mathrm{ic}}\right) & =\frac{1}{2 \pi \overline{\bar{\Theta}^{2}}\left(z_{\mathrm{ic}}\right)} e^{-\frac{r_{\mathrm{ic}}{ }^{2}+r_{\mathrm{w}}{ }^{2}}{2 \overline{\Theta^{2}}\left(z_{\mathrm{ic}}\right)}} \mathrm{I}_{0}\left(\frac{r_{\mathrm{ic}} r_{\mathrm{w}}}{\overline{X^{2}}\left(z_{\mathrm{ic}}\right)}\right) \\
\overline{\theta_{\mathrm{ic}}^{2}}\left(r_{\mathrm{ic}}\right) & =\overline{\Theta^{2}}\left(z_{\mathrm{ic}}\right)-\frac{\overline{X \Theta}^{2}\left(z_{\mathrm{ic}}\right)}{\overline{X^{2}}\left(z_{\mathrm{ic}}\right)}+\left(\frac{\overline{X \Theta}\left(z_{\mathrm{ic}}\right)}{\overline{X^{2}}\left(z_{\mathrm{ic}}\right)}-\frac{1}{L}\right)^{2} r_{\mathrm{w}}{ }^{2} \\
& \times\left[\frac{1}{2}+\frac{\mathrm{I}_{2}\left(\frac{r_{\mathrm{ic}} r_{\mathrm{w}}}{\bar{X}^{2}}\right)}{2 \mathrm{I}_{0}\left(\frac{r_{\mathrm{ic}} r_{\mathrm{w}}}{\bar{X}^{2}}\right)}-\frac{\mathrm{I}_{1}{ }^{2}\left(\frac{r_{\mathrm{ic}} r_{\mathrm{w}}}{\bar{X}^{2}}\right)}{\mathrm{I}_{0}{ }^{2}\left(\frac{r_{\mathrm{ic}} r_{\mathrm{w}}}{\bar{X}^{2}}\right)}\right]
\end{aligned}
$$

where $X$ and $\Theta$ are position and angle projected on the $x$ axis for a particle, $r_{\mathrm{w}}$ is the wobbling radius on the isocentre plane and $\mathrm{I}_{n}(x)=\int_{0}^{\pi} e^{x \cos \theta} \cos (n \theta) \mathrm{d} \theta / \pi$ is the modified Bessel function of the first kind.

The injected beam at the wobbling source $\left(z=z_{\text {src }}\right)$ is modelled to be parallel with finite size, namely, $\overline{\Theta^{2}}\left(z_{\mathrm{src}}\right)=\overline{X \Theta}\left(z_{\mathrm{src}}\right)=0$ and $\overline{X^{2}}\left(z_{\mathrm{src}}\right)=\sigma_{\mathrm{src}}^{2}$, where source size $\sigma_{\text {src }}$ for the $190-\mathrm{MeV}$ proton beam of the HIBMC system has been experimentally determined to $7.76 \mathrm{~mm}$ to reproduce the pristine beam size measured at the isocentre in air without wobbling, scattering, modulation, or range shifting.

Variances $\overline{\Theta^{2}}\left(z_{\text {ic }}\right), \overline{X \Theta}\left(z_{\text {ic }}\right)$ and $\overline{X^{2}}\left(z_{\text {ic }}\right)$ in (A.2) and (A.3) are calculated in the Fermi-Eyges theory in analogy with (2)-(5) for transport from the wobbling source through the beam-modifying devices, which are modelled as slabs of materials, down to the isocentre, ignoring wobbling, collimation and materials downstream of the collimator. Since the angular variance stays constant in the absence of scattering, that of the pencil beam at origin in (2) equals to that in (A.3),

$$
\begin{aligned}
& {\overline{\theta^{2}}}_{i}(0)=\overline{\theta_{\mathrm{ic}}^{2}}\left(r_{\mathrm{ic} i}\right) \\
& r_{\mathrm{ic} i}=\left[\left(\frac{L_{\mathrm{x}}}{L_{\mathrm{x}}-L_{\mathrm{c}}} x_{0 i}\right)^{2}+\left(\frac{L_{\mathrm{y}}}{L_{\mathrm{y}}-L_{\mathrm{c}}} y_{0 i}\right)^{2}\right]^{1 / 2}
\end{aligned}
$$

where $r_{\text {ic } i}$ is the off-axis distance of the pencil beam centre on the isocentre plane.

The beam-wobbling model thus analytically gives the phase-space distribution on the pencil-beam generation plane.

\section{References}

Akagi T, Higashi A, Tsugami H, Sakamoto H, Masuda Y and Hishikawa Y 2003 Ridge filter design for proton therapy at Hyogo Ion Beam Medical Center Phys. Med. Biol. 48 N301-12

Akagi T, Kanematsu N, Takatani Y, Sakamoto H, Hishikawa Y and Abe M 2006 Scatter factors in proton therapy with a broad beam Phys. Med. Biol. 51 1919-28 
Brahme A, Lax I and Andreo P 1981 Electron beam dose planning using discrete Gaussian beams. Mathematical background Acta Radiol. Oncol. 20 147-58

Carlsson A, Andreo P and Brahme A 1997 Monte Carlo and analytical calculation of proton pencil beams for computerized treatment plan optimization Phys. Med. Biol. 42 1033-53

Chu WT, Ludewidt BA and Renner TR 1993 Instrumentation for treatment of cancer using proton and light-ion beams Rev. Sci. Instrum. 64 2055-122

Gottshalk B, Koehler AM, Schneider RJ, Sisterson JM and Wagner MS 1993 Multiple Coulomb scattering of $160 \mathrm{MeV}$ protons Nucl. Instrum. Methods Phys. Res. B $\mathbf{7 4} 467-90$

Hogstrom KR, Mills MD and Almond PR 1981 Electron beam dose calculations Phys. Med. Biol. 26 $445-59$

Hollmark M, Uhrdin J, Belkić Dž, Gudowska I and Brahme A 2004 Influence of multiple scattering and energy loss straggling on the absorbed dose distributions of therapeutic light ion beams: I. Analytical pencil beam model Phys. Med. Biol. 49 3247-65

Hong L, Goitein M, Bucciolini M, Comiskey R, Gottschalk B, Rosenthal S, Serago C and Urie M 1996 A pencil beam algorithm for proton dose calculations Phys. Med. Biol. 41 1305-30

Huq MS, Das IJ, Steinberg T and Galvin JM 2002 A dosimetric comparison of various multileaf collimators Phys. Med. Biol. 47 N159-70

ICRU 1993 Stopping powers and ranges for protons and alpha particles ICRU Report 49 (Bethesda, MD: ICRU)

Jette D 1988 Electron dose calculation using multiple-scattering theory. A. Gaussian multiplescattering theory Med. Phys. 15 123-37

Jiang H and Paganetti H 2004 Adaptation of GEANT4 to Monte Carlo dose calculations based on CT data Med. Phys. 31 2811-18

Kanematsu N, Akagi T, Futami Y, Higashi A, Kanai T, Matsufuji N, Tomura H and Yamashita H 1998 A proton dose calculation code for treatment planning based on the pencil beam algorithm Jpn. J. Med. Phys. 18 88-103

Kanematsu N, Matsufuji N, Kohno R, Minohara S and Kanai T 2003 A CT calibration method based on the polybinary tissue model for radiotherapy treatment planning Phys. Med. Biol. 48 1053-64

Kohno R, Kanematsu N, Yusa K and Kanai T 2004 Experimental evaluation of analytical penumbra calculation model for wobbled beams. Med. Phys. 31 1153-57

Komori M, Furukawa T, Kanai T and Noda K 2004 Optimization of Spiral-Wobbler System for Heavy-Ion Radiotherapy Jpn. J. Appl. Phys. 43 6463-7

Oozeer R, Mazal A, Rosenwald JC, Belshi R, Nauraye C, Ferrand R and Biensan S 1997 A model for the lateral penumbra in water of a $200-\mathrm{MeV}$ proton beam devoted to clinical applications. Med. Phys. 24 1599-604

Paganetti H 1998 Monte Carlo method to study the proton fluence for treatment planning Med. Phys. $252370-75$

Paganetti H, Jiang H, Lee SY and Kooy HM 2004 Accurate Monte Carlo simulations for nozzle design, commissioning and quality assurance for a proton radiation therapy facility Med. Phys. $312107-18$

Petti PL 1992 Differential-pencil-beam dose calculations for charged particles Med. Phys. 19 137-49

Russell KR, Isacsson U, Saxner M, Ahnesjö A, Montelius A, Grusell E, Dahlgren CV, Lorin S and Glimelius B 2000 Implementation of pencil kernel and depth penetration algorithms for treatment planning of proton beams Phys. Med. Biol. 45 9-27

Schaffner B, Pedroni E and Lomax A 1999 Dose calculation models for proton treatment planning using a dynamic beam delivery system: an attempt to include density heterogeneity effects in the analytical dose calculation Phys. Med. Biol. $4427-41$

Suit H and Urie M 1992 Proton beams in radiation therapy J. Natl. Cancer Inst. 84 155-64

Szymanowski H, Mazal A, Nauraye C, Biensan S, Ferrand R, Murillo MC, Caneva S, Gaboriaud G and Rosenwald JC 2001 Experimental determination and verification of the parameters used in a proton pencil beam algorithm Med. Phys. 28 975-87

Tomura H, Kanai T, Higashi A, Futami Y, Matsufuji N, Endo M, Soga F and Kawachi K 1998 Analysis of the penumbra for uniform irradiation fields delivered by a wobbler method Jpn. J. Med. Phys. 18 42-56

van Luijk P, van't Veld AA, Zelle HD and Schippers JM 2001 Collimator scatter and 2D dosimetry in small proton beams. Phys. Med. Biol. 46 653-67 